\title{
On the degree of attention and capacity limitations in visual processing*
}

\author{
RICHARD M. SHIFFRIN $\dagger$, GERALD T. GARDNER $\dagger+$, and DAVID H. ALLMEYER \\ The Rockefeller University, New York, New York 10021
}

\begin{abstract}
The ability to detect simple types of visual information from various locations in the visual field was examined in two experiments. In both, the four locations containing the information to be detected were presented either simultaneously or sequentially, with the presentation time per location identical for both conditions. Limitations of capacity and attentional control of perceptual processing would predict sequential presentation to be superior to simultaneous presentation, since, in the former case, attention need not be shared among four locations. The results showed equal detection performance for both conditions. Thus, spatial attention was not present during detection of horizontal and vertical dot pairs (Experiment 1 ). $O$ differences (probably due to strategies) make the same conclusion more difficult to draw for single-dot detection (Experiment II). Similar findings and conclusions were reported by Shiffrin and Gardner (1972) in studies utilizing alphabetic characters.
\end{abstract}

The questions of attentional control and limitations of capacity in sensory information processing are among the most basic in psychology. Figure 1 presents three of the commonly proposed models of sensory processing. The top panel illustrates a single-channel model in which information enters the system from only a single source at any one moment. It is assumed in this model that attention is switched rapidly among various possible sources of sensory information. Models similar to this were originally proposed by Broadbent (1958) in the context of dichotic listening experiments. Franzen, Markowitz, and Swets (1970) proposed a model of this type for the processing of near-threshold vibrotactile information. Estes and Taylor $(1964,1966)$ proposed a model of this type for visual processing. A less extreme attentional model is shown in the middle panel of the figure. In this attenuation model, total processing capacity is limited, but some input is simultaneously or serially processed from many channels. The O's attentional control determines the amount of information processed in particular channels. Treisman (1969), Moray (1970), and Neisser (1967) have proposed models of this type with evidence primarily based on dichotic listening and other auditory experiments. Rumelhart (1970) and Norman and Rumelhart (1970) have proposed a model of this type based primarily on experimentation in visual processing. It should be noted that both types of limited-capacity attentional models require a "preattentive" mechanism to direct attention to important channels (see Neisser, 1967 , for a discussion of the need for preattention in these models). The final class of model is seen in the lower panel of Fig. 1. This model assumes minor limitations of capacity and no attention during

*This research was supported by PHS Grant $12717-06$ to the first author. The authors are indebted to Professor William $\mathrm{K}$. Estes, who donated the use of his laboratory to carry out the experiments.

+ Requests for reprints should be sent to Richard M. Shiffrin, now at the Department of Psychology. Indiana University, Bloomington. Indiana 47401

++ Now at the University of Michigan at Dearborn. perceptual processing. All attentional effects are due to characteristics of short-term store following perceptual processing. Deutsch and Deutsch (1963) and Deutsch, Deutsch, and Lindsay (1967) have proposed such a model for auditory processing. Gardner $(1970,1973)$, Shiffrin and Gardner (1972), and Shiffrin and Geisler (in press) have proposed this model for visual processing and general sensory processing; Shiffrin, Craig, and Cohen (in press) proposed such a model for tactile processing.

The Shiffrin and Gardner (1972) result is particularly important because it places clear and severely limited bounds on the degree of attention which is present in visual processing. Their result argued strongly for a model of the nonattention type, the lower model in Fig. 1. The paradigm was based in part upon an earlier one by Eriksen and Spencer (1969) and tests the ability
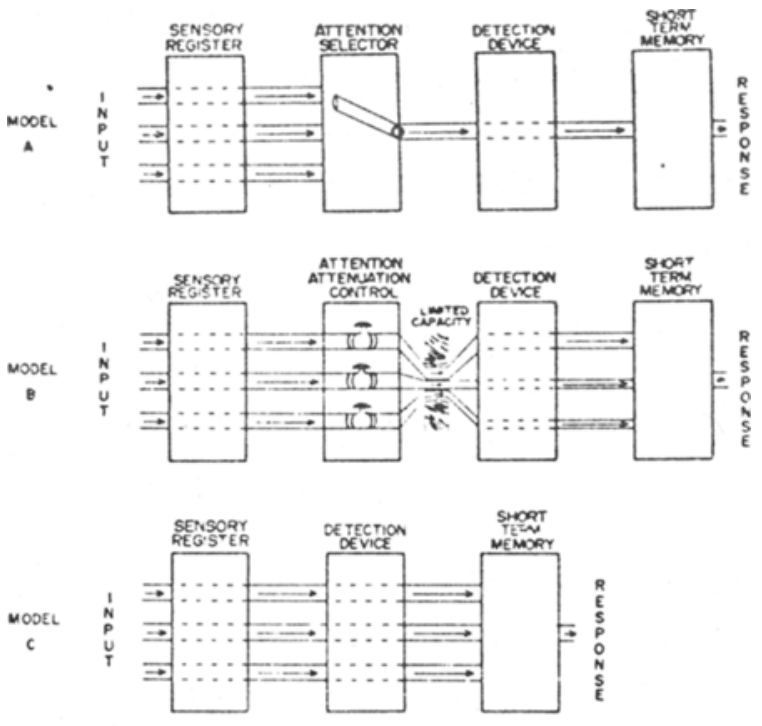

SENSORY PROCESSING MODELS

Fig. 1. Models for the initial stages of sensory information processing. (A) Single-channel limited-capacity attentional model: (B) multichannel limited-capacity attentional model: (C) parallel unlimited-capacity nonattentional model. 
to distribute attention across spatial locations. The $\mathrm{O}$ is asked to say which of two critical letters ( $T$ or $F$ ) is present in a display of four elements. Three of these elements are distractors, and their degree of confusability with the critical elements is varied; the remaining element is one of the two critical elements. Each element is presented for $t_{1}$ msec (about 40), preceded and followed by masks. There are two key conditions. In the first, called simultaneous, the four elements are presented simultaneously. In the second, called sequential, the four letters are presented sequentially. In the simultaneous condition, the $\mathrm{O}$ must share his "attention" among the four locations; in the sequential condition, the $O$ can give all his "attention" to each letter as it appears. Despite this, in three experiments, the sequential and simultaneous conditions gave equal performance.

The conclusions from the Shiffrin and Gardner experiment are limited in several ways. First, only four display elements were used. It is possible that limitations of capacity and attention would be seen if more than four elements were utilized. Secondly, the experiment in question used the same stimulus materials throughout: the critical letters were $T$ and $F$, and the distractors were either a zero ( 0 ) or a T-F hybrid ( 7$)$. Perhaps the results apply only when letters are used and would differ if a different detection task was utilized.

The present experiment explores variations in the types of stimuli used. In particular, we ask whether simpler detection tasks, nonalphanumeric in character, would give rise to attentional effects. This extension is important because it is sometimes proposed that attentional effects will occur only during detection of simple near-threshold stimuli (see Franzen, Markowitz, \& Swets, 1970). In Experiment I, we utilize critical elements consisting of horizontal and vertical dot pairs, with distractors being either diagonal dot pairs or a single dot. In Experiment II, we ask for detection of the position of a single dot in one of the four locations.

\section{EXPERIMENT I}

\section{Method}

\section{Observers}

One male and five female college-age Os were paid $\$ 4$ for each $2-h$ session. The Os had answered a newspaper ad, and three of them had served in other tachistoscopic experiments in the laboratory.

\section{Stimuli and Equipment}

Stimuli consisted of dot patterns displayed on the face of a cathode ray tube (CRT) driven by a PDP-12 computer. An array of four of these dot patterns appears on the screen at a given moment. One of the four dot patterns was the "target" and was either a ":" or an ".."; the remaining "noise" characters were all single dots in the low-confusability condition and diagonal dot pairs in the high-confusability condition. All four dot patterns that were used are shown in Fig. 2. Each stimulus appeared at one corner of an imaginary square, with adjacent stimuli separated by a minimum visual angle of $1.5 \mathrm{deg}$. A fixation dot subtending $0.02 \mathrm{deg}$ was displayed at all times at the center of the square. A given pattern subtended a maximum of $14 \mathrm{~min}$ vertically and 9 min horizontally.

Although the CRT was equipped with P-31 fast-decay phosphor, the time elapsed between successive replottings of the array was less than 300 -microsec, so that no flicker was visible. In addition, the time between successive replottings was held constant, regardless of experimental condition. Luminous intensity per dot was approximately $7 \times 10^{-6} \mathrm{~cd}$ (see Sperling, 1971), and overhead illumination in the experimental room resulted in a .8-fL luminance for background portions of the tube.

The $O$ sat at the head of a black slanted panel used to minimize reflections from the CRT screen. As a result, $O$ 's eyes were placed at a viewing distance of $87 \mathrm{~cm}$ from the fixation dot and were raised about $10 \mathrm{~cm}$ above the fixation dot. Responses and trial starts were made on a labeled set of microswitch panels placed directly in front of $\mathrm{O}$ below the reflection shield.

The main independent variable was the temporal mode of presentation of the individual stimuli of an array. In the simultaneous condition (SIM), all four stimuli appeared at the same time; in the sequential condition (SEQ), only one stimulus was displayed at a time. The top diagram of Fig. 3 illustrates a typical stimulus exposure in the SIM condition: (1) the fixation point appeared continuously until $O$ depressed a microswitch panel. When this occurred, (2) four masking patterns were displayed for $400 \mathrm{msec}$, one pattern at each of the four corner positions; each pattern consisted of a 4 by 6 dot matrix. (3) Following these "premasks," the four stimuli appeared for a preselected exposure duration, which was determined individuaily for each $O$ (see below). (4) Immediately after the offset of the stimulus array, four postmasks, identical to the premasks, were displayed continuously until $O$ made his responses. The bottom diagram of Fig. 3 illustrates a typical stimulus exposure in the SEQ condition. In the description that follows, the upper-left and lower-right comers of the array are together referred to as "Diagonal A," and the upper-right and lower-left corners as "Diagonal B." (1) The fixation point appeared continuously until the $O$ 's start signal, at which time (2) four premasks were displayed; $400 \mathrm{msec}$ after the onset of the premasks, the two premasks in one diagonal, Diagonal $A$ in the illustration, were replaced by stimuli, which then remained on for the same exposure duration used in the simultaneous condition. (3) After this duration had elapsed, the two stimuli were replaced by postmasks and four masks remained on the screen for $500 \mathrm{msec}$. (4) After this 500 -msec period had elapsed, the premasks in the other diagonal (in this case, Diagonal B) were replaced by the remaining two stimuli. The second pair of stimuli remained on for the preselected duration, and (5) were

\begin{tabular}{|c|c|c|}
\hline $\begin{array}{llll}0 & 0 & 0 & 0 \\
0 & 0 & 0 & 0 \\
0 & 0 & 0 & 0 \\
0 & 0 & 0 & 0 \\
0 & 0 & 0 & 0 \\
0 & 0 & 0 & 0\end{array}$ & $\begin{array}{llll}0 & 0 & 0 & 0 \\
0 & 0 & 0 & 0 \\
0 & 0 & 0 & 0 \\
0 & 0 & 0 & 0 \\
0 & 0 & 0 & 0 \\
0 & 0 & 0 & 0\end{array}$ & $\begin{array}{llll}0 & 0 & 0 & 0 \\
0 & 0 & 0 & 0 \\
0 & 0 & 0 & 0 \\
0 & 0 & 0 & 0 \\
0 & 0 & 0 & 0 \\
0 & 0 & 0 & 0\end{array}$ \\
\hline "LOW" & "HIGH" & VERTICAL \\
\hline $\begin{array}{l}\text { CONFUSABLE } \\
\text { NOISE }\end{array}$ & $\begin{array}{l}\text { CONFUSABLE } \\
\text { NOISE }\end{array}$ & $\begin{array}{l}\text { TARGET } \\
\text { PATTERN }\end{array}$ \\
\hline PATTERN & PATTERN & \\
\hline \multicolumn{3}{|c|}{ STIMULUS PATTERNS } \\
\hline & \multicolumn{2}{|c|}{$\begin{array}{c}\text { FOR EXPERIMENT I } \\
\text { (DIAGONALS) }\end{array}$} \\
\hline
\end{tabular}

Fig. 2. Stimulus patterns for Experiment I (filled circles represent points illuminated on the CRT screen).

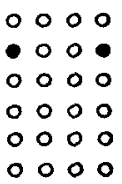

HORIZONTAL TARGET PATTERN 
Fig. 3. Display procedure for Experiment I. Each row in the figure gives the temporal sequence for a single trial in the experiment.

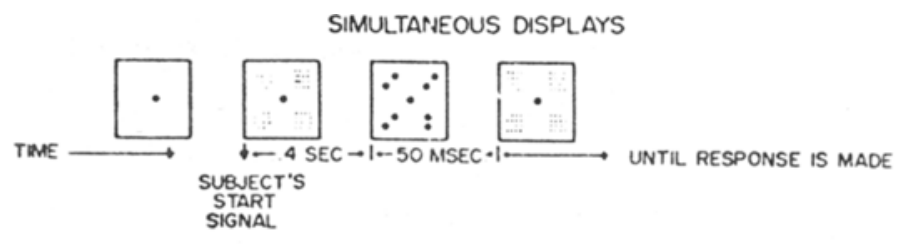

\section{SEQUENTIAL DISPLAYS}
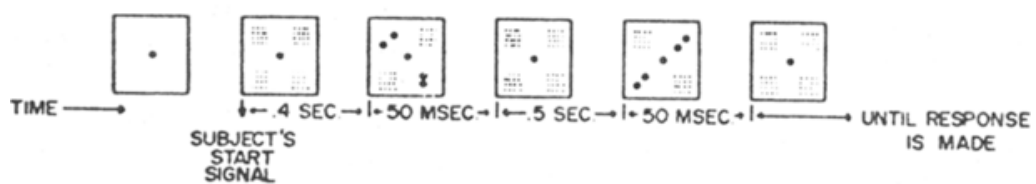

\section{EXPERIMENT I (DIAGONALS)}

then replaced by postmasks. The full set of four postmasks remained on until $\mathrm{O}$ made his responses.

\section{Procedure}

Each $O$ served in an initial practice session and three experimental sessions. All sessions involved the same basic procedure. On every trial, $O$ centered his gaze on the fixation dot, and initiated the stimulus exposure by pressing a microswitch panel. Following the exposure, $O$ indicated whether the array contained a ":" or an ". "by depressing one of two panels and then indicating what corner location the target had appeared in by depressing one of four additional panels. A complete replication of the experimental design involved 64 trials-four 16-trial blocks, successive blocks alternating between SIM and SEQ conditions. Marker stimuli appeared before each block to indicate the presentation condition for that block. One of the SEQ blocks involved arrays in which Diagonal $A$ appeared first, the other $S E Q$ block involved arrays in which Diagonal $B$ appeared first. The marker stimuli appearing before the SEQ blocks indicated the order of appearance of the diagonals.

Within each of the four 16-trial blocks, half of the trials involved high-confusability arrays and half involved low-confusability arrays. For both types of array, ":" and ".." were used as target stimuli equally often and appeared at each possible spatial location with equal frequency. The level of confusability, choice of target stimulus. and location of target stimulus all varied randomly within a block. The Os were permitted to work at their own pace, and to complete as many 64-trial replications as they could without fatigue during each 2-h session; they were urged to stop and rest as often as necessary to avoid fatigue. The number of blocks completed per session varied across Os from approximately 30 to 50 .

During the practice session, Os were familiarized with the experimental procedure. They received trial-by-trial feedback on the first four 16-trial blocks; feedback thereafter was only given informally at the end of the experimental sessions. Additional practice session trials were then used to determine a stimulus exposure duration for each $\mathrm{O}$ that yielded $80 \%$ accuracy in detecting the target stimuIi in low-confusability SIM arrays. The duration was determined by a modified up-and-down method, and was altered occasionally during experimental sessions in order to maintain the $80 \%$ level despite changes in the degree of practice. These exposure duration changes, however. occurred only between complete. successive replications of the experimental design. The average exposure duration over all sessions is given for each $O$ in the lowest row of $T$ able 1 .

The first two 16-trial blocks of each experimental session served as a warm-up period, and the resulting data were not included in the summaries and analyses reported below.

\section{Results}

The main features of the data are presented in Tables $1 \mathrm{a}, 1 \mathrm{~b}$, and $1 \mathrm{c}$, which give the results both for individual Os and for the group average. The table includes the data for the simultaneous and sequential conditions within each level of confusability, and also averaged over confusability. The lowest horizontal row (difference) gives the difference between simultaneous and sequential conditions in each case, a positive number indicating an advantage for the simultaneous condition. Table la gives the probability of correct detection of a horizontal or vertical target stimulus. Table $1 b$ gives the identical table, except that the entries are the probability of a correct location judgment, and Table $1 \mathrm{c}$ gives the identical table, except that the entries are the probability of a jointly correct judgment of location and stimulus type.

The data are summarized by the lower-right-hand corner of each of Tables $1 \mathrm{a}, \mathrm{lb}$, and $1 \mathrm{c}$. This cell gives the overall difference between simultaneous and sequential conditions. A repeated-measures analysis of variance (Lindquist, 1953) confirmed what is evident from the table: mode of presentation yielded no significant difference for any of the three measures. Confusability had a small, but significant, effect on the stimulus and location measures, but no significant difference for the correct-on both measure $[F(1,5)=$ $9.60, p<.05 ; F(1,5)=12.40, p<.05 ;$ and $F(1,5)=$ 2.35 , n.s.. respectively]. Of the remaining variables and interactions, the only significant effects of interest concern the $O$ variable. This variable was significant on all three measures. There was an interaction of Os with mode of presentation: this interaction was significant for each of the three measures $[F(1.5)=7.7, p<.05 ; F(1,5)$ $=13.3, p<.05: F(1.5)=10.7, p<.05$. respectively $]$. Thus, although simul taneous and sequential presentation 
Table 1

Results of Experiment I

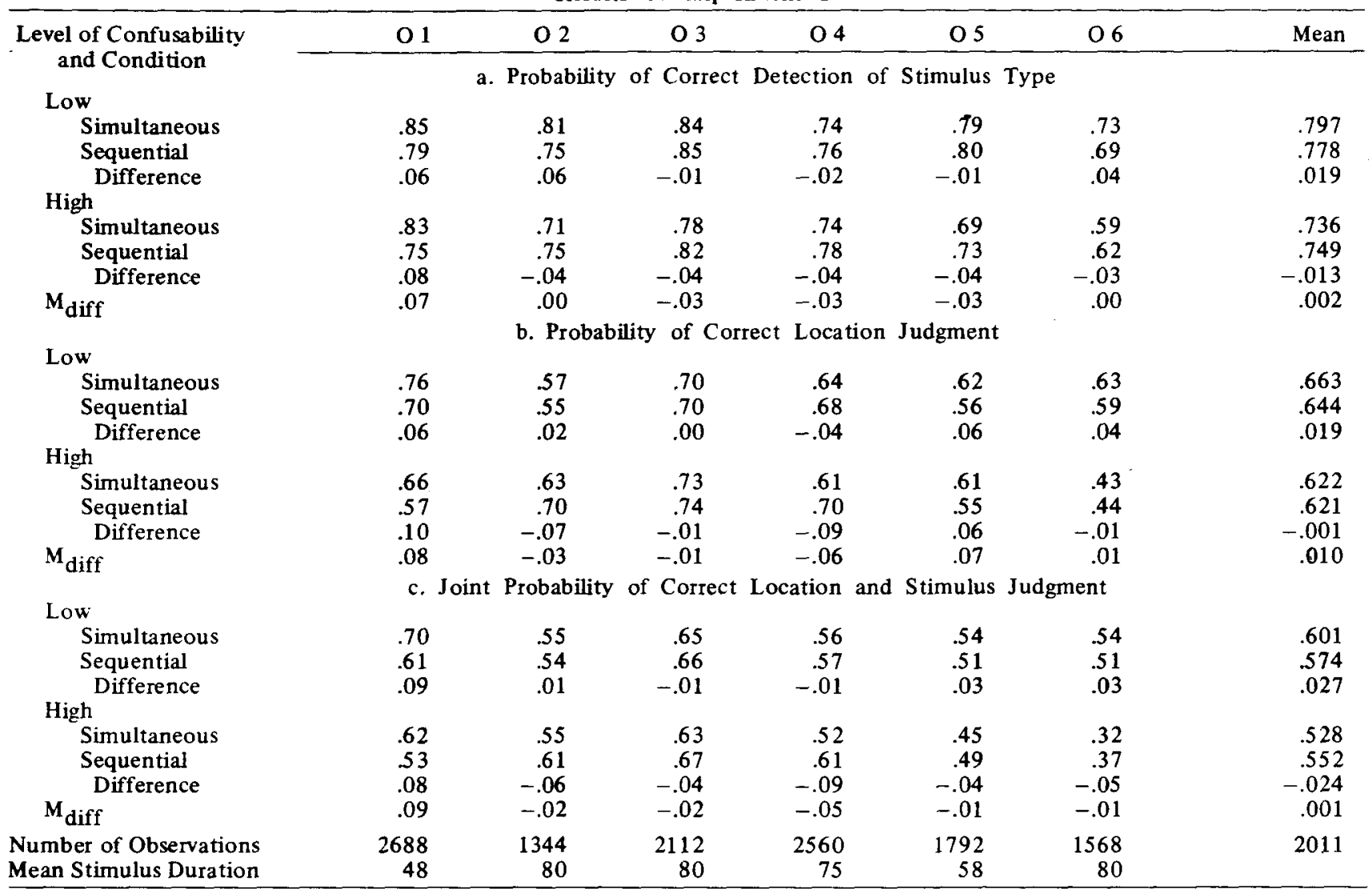

did not differ for the group, the difference between these conditions was significant for some Os. Actually, four Os showed no significant difference, and two Os showed a difference, one in favor of each presentation mode.

\section{Discussion}

This experiment was consistent with the three reported in Shiffrin and Gardner (1972) in showing no difference between sequential and simultaneous conditions. Hence, the failure to find attentional effects apply to simple dot stimuli as well as to alphabetic characters. In this experiment, there was relatively little difference between the distractors thought in advance to be of high and low confusability. As shown in previous studies, confusability will affect overall performance but not interact with presentation mode; in the present study, it appears that the distractors were of nearly equal confusability, but this fact in no way affects the primary conclusion. The significant differences among Os is not important, since the levels were set for each $O$. However, the interaction of Os with presentation mode is worthy of consideration. Apparently, one $\mathrm{O}$ was superior on sequential trials and one $O$ was superior on simultaneous trials. Although the effects were small, none of the theories (including the nonattention model) discussed so far make provision for such deviations. It is tempting to suggest that differing decision strategies could account for the differences observed, but careful questioning of Os did not reveal any evident differential strategies; nor do we have any strategies to suggest which can be supported by independent evidence. It is, nevertheless, possible that such strategies are responsible for the effects. For example, some Os could have a poor short-term retention strategy, causing them to forget between phases of the sequential trials and thereby giving a slight advantage for the simultaneous conditions. Other Os could have excellent retention, but adopt a strategy of ignoring relevant information on certain channels during simultaneous presentation, thereby giving sequential trials a slight advantage.

In summary, we should keep in mind that $O$ differences are not vary large, and should not affect the central conclusions. Thus, this experiment does argue strongly that attentional effects and limitations of processing capacity are very small at best. To extend these conclusions to even simpler stimuli than were used in the present experiment, we carried out a final experiment in which the task was detection of the location of a single dot. 
Fig. 4. Display procedure for Experiment II. Each row in the figure gives the temporal sequence for a single trial in the experiment.
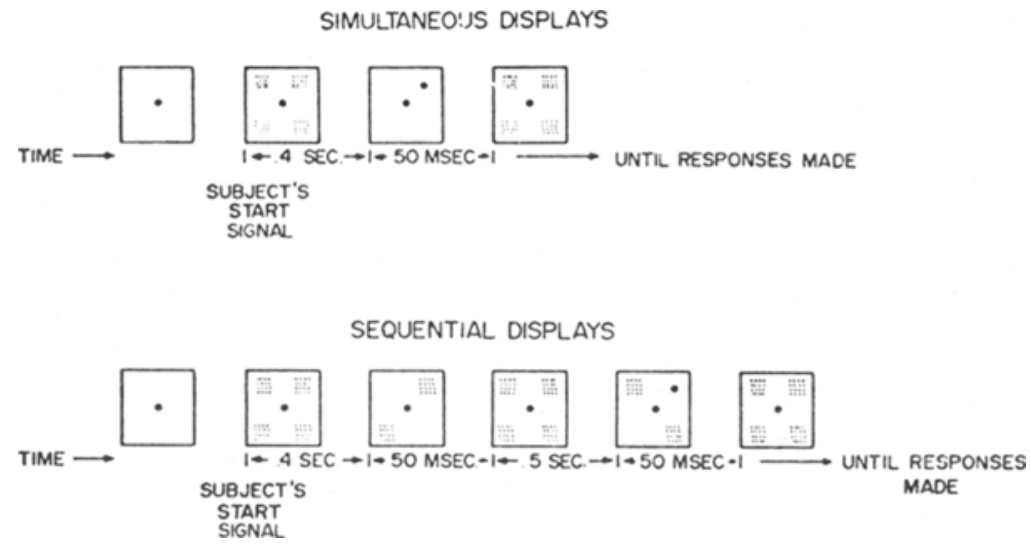

\section{EXPERIMENT II}

\section{Method}

\section{Observers}

Five of the six Os who served in Experiment $I$ also served in Experiment II. O 6, female, could not continue, leaving four females and one male.

\section{Stimuli and Equipment}

The target stimulus, a single dot, held a given location with respect to the masking stimulus that followed. The dot occupied the position within the middle four positions of the 4 by 6 mask array that was closest to the fixation point. The O's sole task was to estimate the location of the single dot among the four display positions. The equipment and other details were identical to those of Experiment 1

\section{Procedure}

The procedure is illustrated in Fig. 4. The $O$ guessed the position of a single dot-there were no distractors. The exposure duration was set so that Os responded correctly on about $65 \%$ of the trials (chance guessing would result in $25 ?$ probability correct). All randomizations and other procedural details are the same as in Experiment I.

\section{Results}

The main results are given in Table 2 . An analysis of variance like that of Experiment I revealed no significant effect of mode of presentation, but a highly significant effect of $\mathrm{Os}$ and of an $\mathrm{O}$ by Mode of Presentation interaction $[F(1,4)=11.8, p<.05 ; F(1,4)=24.9$, $\mathrm{p}<.01$, respectively). In this experiment, the effects of $\mathrm{O}$ differences are marked, some Os showing an advantage for simultaneous and the others showing an advantage for sequential presentations. One might be tempted to attribute the large advantage of simultaneous presentation (in the two Os who exhibited this) to some lack of motivation or other O-controlled effect, but for the fact that these two Os were the best in all respects. They maintained the highest aceuracy at the lowest exposure durations and did this while operating at the highest speeds per trial.

In the series of five experiments in vision (the first three from Shiffrin \& Gardner, 1972), this experiment is the only one showing large $O$ differences. One might argue that the cause lies in the simplicity of the task-simple detection with no distractors. However, in the Shiffrin, Craig, and Cohen (in press) study, the same paradigm involving tactile detection revealed no large $O$ differences or interactions.

It is interesting to note that the pattern of $\mathrm{O}$ differences in this experiment corresponded to the differences in Experiment I seen in the location judgment measure. However, they have become accentuated in the present study. If the Os were beginning to use strategies in Experiment $I$, these tendencies could have greatly increased in the present paradigm. The possibility of the use of fixation strategies is accentuated in the detection paradigm due to the effect of acuity gradients. It is possible that stimulus duration could be set so that central fixation would result in extremely low detectability while fixation on one of the four stimulus locations would result in high detectability whenever that location happened to contain a signal. This possibility presents an opportunity for a variety of fixation strategies, perhaps varying according to presentation mode. A look at the fine-grained breakdown of the data reveals numerous deviations (as, say, a function of spatial position) that

Table 2

Results of Experiment II

\begin{tabular}{lrrrrrr}
\hline Condition & $\mathrm{O} 1$ & $\mathrm{O} 2$ & $\mathrm{O} 3$ & $\mathrm{O} 4$ & $\mathrm{O} 5$ & Mean \\
\hline $\begin{array}{l}\text { Simultaneous } \\
\text { Sequential }\end{array}$ & .65 & .59 & .65 & .64 & .81 & .671 \\
Difference & .12 & -.09 & -.68 & .71 & .62 & .637 \\
$\begin{array}{l}\text { Number } \\
\text { of Trials }\end{array}$ & 2496 & 1920 & 1664 & 2304 & 2336 & 2145 \\
$\begin{array}{l}\text { Mean Expo. } \\
\text { sure Duration }\end{array}$ & 48 & 60 & 59 & 67 & 53 & .07 \\
\hline
\end{tabular}


might reflect various strategies by different Os. The patterns do not appear to be consistent enough to let us make a case for a particular fixation strategy, and we do not intend to do so. Nevertheless, the possibility of such strategies suggests that visual detection paradigms, like those of Experiment II, had better be carried out with the aid of eye-movement monitors in the future.

Although the lack of an average advantage for SEQ over SIM might make us wish to conclude that attention is inoperative, the wide $\mathrm{O}$ differences make any conclusion very weak.

The results of Experiment I say that information on four visual channels can be encoded and evaluated as efficiently when all four channels occur simultaneously as when only two at a time occur simultaneously. If attention was operative, it could have been switched between successive diagonals, leading to superior performance in the SEQ condition. Hence, we conclude that attention was not operative in that situation. This result and conclusion supports those reached in Shiffrin and Gardner (1972), using alphanumeric-type stimuli, and those reached by Shiffrin, Craig, and Cohen (in press) for tactile stimuli.

\section{REFERENCES}

Broadbent, D. E. Perception and communication. London: Pergamon, 1958.

Deutsch, J., \& Deutsch, D. Attention: Some theoretical considerations. Psychological Review, 1963, 70, 80-90.

Deutsch, J., Deutsch, D., \& Lindsay, P. Comments on 'Selective attention: Stimulus of response.' Quarterly Journal of Experimental Psychology, 1967, 19, 362-368.

Eriksen, C. W., \& Spencer, T. Rate of information processing in visual perception: Some results and methodological considerations. Journal of Experimental Psychology Monograph, 1969, 79, 1-16.

Estes, W. K., \& Taylor, H. A. A detection method and probabilistic models for assessing information processing from brief visual displays. Proceedings of the National Academy of
Sciences of the United States of America, 1964, 52, 446-454.

Estes, W. K., \& Taylor, H. A. Visual detection in relation to display size and redundancy of critical elements. Perception \& Psychophysics, 1966, 1. 9-16.

Franzen, O., Markowitz, J., \& Swets, J. A. Spatially-limited attention to vibrotactile stimulation. Perception \& Psychophysics, 1970, 7, 193-196.

Gardner, G. T. Spatial processing characteristics in the perception of brief visual arrays. (Doctoral dissertation) Technical Report No. 23, Human Performance Center, University of Michigan, 1970.

Gardner, G. T. Evidence for independent parallel channels in tachistoscopic perception. Cognitive Psychology, 1973, 4, 130-155.

Lindquist, E. F. Design and analysis of experiments in psychology and education. Boston: Houghton-Mifflin, 1953.

Moray, N. Attention: Selective processes in vision and hearing. New York: Academic Press, 1970.

Neisser, U. Cognitive psychology. New York: Appleton-Century-Crofts, 1967.

Norman, D. A., \& Rumelhart, D. E. A system for perception and memory. In D. A. Norman (Ed.), Models of human memory. New York: Academic Press, 1970.

Rumelhart, D. E. A multicomponent theory of the perception of briefly exposed visual displays. Journal of Mathematical Psychology, 1970, 7, 191-218.

Shiffrin, R. M., Craig, J. C., \& Cohen, U. On the degree of attention and capacity limitations in tactile processing. Perception \& Psychophysics, 1973, 13, 328-336.

Shiffrin, R. M., \& Gardner, G. T. Visual processing capacity and attentional control. Journal of Experimental Psychology. $1972,93,72-82$.

Shiffrin, R. M., \& Geisler, W. S. Visual recognition in a theory of information processing. In $\mathrm{R}$. Solso (Ed.), The Loyola symposium: Contemporary viewpoints in cognitive psychology. Washington: Winston, in press.

Sperling, G. The description and luminous calibration of CRO displays. In N. Moray (Ed.), On-line computing for behavioral science. Brussels: NATO Institute, in press.

Treisman, A. Strategies and models of selective attention. Psychological Review, 1969, 76, 282-299.

(Received for publication February 26, 1973; revision accepted A pril 6,1973 .) 